\title{
Biases in Amino Acid Replacement Matrices and Alignment Scores Due to Rate Heterogeneity
}

\author{
Colleen Kelly* \\ Department of Computer Science and Statistics \\ University of Rhode Island, Kingston, RI 02881 \\ phone: (401)792-2701_email: kelly@stat.uri.edu \\ Gary A. Churchill \\ Department of Biometry and Plant Breeding \\ Cornell University, Ithaca, NY 14853
}

Running Head: Bias in Replacement Matrices

Keywords: rate variation, protein alignment, PAM matrix, evolutionary distance.

${ }^{0}$ To whom correspondence should be addressed. 


\begin{abstract}
Empirically derived amino-acid replacement matrices are widely used in sequence comparison and database searches. We consider an extension of the usual Markov process model of protein evolution that admits site to site rate heterogeneity and demonstrate that rate heterogeneity can introduce a bias in estimated replacement probabilities and the corresponding alignment scores derived from these matrices. We suggest an approach to obtain unbiased estimates of replacement probabilities and alignment scores and derive the details for the case where rates are assumed to vary according to a Gamma distribution.
\end{abstract}




\section{Introduction}

Empirically derived amino acid replacement matrices (Dayhoff et al. 1978, Jones at al. 1992, Gonnet et al. 1992) are widely used in problems of sequence comparison and alignment (Altschul 1991, 1993) and in database searches (Altschul et al. 1990). Replacement matrices reflect the average (over many sites in many protein families) probabilities with which one amino acid may be substituted by another over evolutionary time. Because they are empirically derived, they should reflect exchangeability due to physical and chemical similarities of amino acids as well as effects due to properties of the underlying genetic code and the mutation processes acting at the DNA level. Empirically derived matrices are generally considered to reflect the true relationships among amino acids better than matrices derived from considerations of chemical or physical properties (Taylor 1986) or the genetic code (Feng and Doolittle 1985).

The pioneering work of Dayhoff and her collaborators (Dayhoff et al. 1978) has recentiy been updated to include the large arnounts of protein sequence information that have accumulated since 1978 (Jones et al. 1992, Gonnet et al. 1992). The general pattern of the replacement probabilities appears to be remarkably stable in spite of the rather limited set of protein families that were available then as compared to the present (Jones et al. 1992). This suggests that it may be reasonable to use average replacement matrices although any particular family (or site within a family) may have its own characteristic pattern of replacements. It may be possible to develop a small set of distinct replacement matrices (Sander and Schneider 1991, Brown et al. 1993) that reflect different local environments within proteins or different protein families. If this is the case, the methods described here will continue 
to be useful in deriving appropriate unbiased estimates of the replacement probabilities.

The model of sequence change which is usually assumed in the construction of amino acid replacement matrices is a Markov process model that describes the pattern of replacements over time and acts independently and identically at each residue of a protein sequence. A critical discussion of this model is given by Wilbur (1985) who raises a number interesting points.

It is commonly accepted and evident from observation that rates of change are not identical at each site in a sequence (Uzzell and Corbin 1971, Holmquist 1983, Reeves 1992, Wakely 1993, Yang et al. 1994). Rate variation is probably present to a greater or lesser extent in every protein family. We are of the opinion that site to site rate variation presents one the most important and challenging problems now faced by methodological researchers in molecular evolution and that new methods will be needed by empirical scientists to properly analyze and interpret sequence data. It has already been observed that rate variation can introduce biases into estimates of sequence divergence (Kelly 1991, Kelly and Rice 1994, Yang et al. 1994, Ota and Nei 1994). We take the point of view that the Markov model is an acceptable approximation to the actual process of sequence evolution at any one site and address the issue of estimating an average replacement matrix in the presence of rate heterogeneity.

Clearly, any one pair of protein sequences will not provide sufficient information to estimate the large number of parameters required to specify a complete replacement matrix. Thus, many such pairs or families of sequences must be considered. A problem arises because not all sequence families are evolving at the same rates and not all pairs of sequences are separated by similar amounts of geological time. Thus to obtain estimates of replacement 
probabilities over a given interval of evolutionary time $t$ (geological time $\times$ rate of evolution), the observed patterns of replacements must be adjusted to reflect a common amount of divergence.

Dayhoff et al. (1978) proposed measuring time in PAM units. One PAM unit corresponds to an average of one replacement per 100 sequence residues for a protein of average composition. In the past, it has been common to use the so called "PAM250" log-odds scoring matrix for sequence alignment and database searches. However, this matrix was developed for detecting very distant relationships (Schwartz and Dayhoff, 1978). It has been pointed out (Karlin and Altschul 1991) that the choice of a scoring matrix implies a particular target distribution of aligned amino acid pairs and that optimal results are obtained when the scoring matrix corresponds to the evolutionary distance between the particular sequences being compared. Altschul (1993) recommends the use of a small set of scoring matrices tuned to distinct evolutionary distances for database searching applications.

Replacement matrices at different PAM distances are typically computed by repeated mulitplication of a PAM1 matrix. We have noticed that there is a bias, due to rate heterogeneity, introduced into rescaled replacement matrices computed by this method. We describe the nature of this bias and suggest an adjustment in the case where rates are assumed to follow a Gamma distribution with known shape parameter $\alpha$. In section 2 of this paper, we review the basic concepts of Markov models of sequence evolution for the case of homogenous rates and describe an extension to the heterogeneous rates case. We then briefly review the calculation of alignment scores from replacement matrices. In section 3 we demonstrate that the standard method of adjustment introduces a bias in both the replacement probabilities and their corresponding alignment scores. In section 4 we demonstrate these biases using data from the 
blocks data base (Henikoff and Henikoff 1991). In the final section, we discuss some of the consequences of rate heterogeneity on the estimated replacement rates. 


\section{Methods}

\subsection{A Homogeneous Rates Model}

We assume that the process of amino acid replacement acting at one site can be described as a continuous time, time homogeneous Markov process. We further assume that the process is reversible in time and that it is has reached its equilibrium state. The reader is referred to Tavaré (1986) for a detailed description and criticisms of this model for the case of nucleotide sequences.

Under the Markov model, a site originally occupied by amino acid $i$ will, after (evolutionary) time $t$, be occupied by amino acid $j$ with probability $p_{i j}(t)$, for $i=1, \ldots, 20$ and $j=1, \ldots, 20$ where $1, \ldots, 20$ is an arbitrary numbering of the amino acids. The matrix of transition probabilities may be written

as $\mathbf{P}(t)=\left[p_{i j}(t)\right]$. Such transition matrices are commonly expressed as the matrix exponential of a rates matrix $\mathbf{Q}$. Let $\mathbf{Q}=\mathbf{U}^{-1} \Sigma \mathbf{U}$ be the spectral decomposition of $\mathbf{Q}$. We can express $\mathbf{P}(t)$ as

$$
\begin{aligned}
\mathbf{P}(t) & =\epsilon^{\mathbf{Q} t} \\
& =\mathbf{U}^{-1} e^{\Sigma t} \mathbf{U} \\
& =\mathbf{U}^{-1} \boldsymbol{\Theta}(t) \mathbf{U}
\end{aligned}
$$

where $\mathbf{U}$ is a matrix whose columns are the eigenvectors of $\mathbf{Q}, \Sigma$ is a diagonal matrix of eigenvalues, $\Sigma=\operatorname{diag}\left\{\sigma_{1}, \ldots, \sigma_{20}\right\}$ and $\Theta(t)=\operatorname{diag}\left\{e^{\sigma_{1} t}, \ldots, e^{\sigma_{20} t}\right\}$. This form is especially convenient for computing transition matrices at various times $t$.

If the spectral decompostion of $\mathbf{Q}$ can be obtained, the transition probability matrix can be adjusted to any other time $\tau$ by a mapping of the eigenvalues

$$
\sigma_{i} \rightarrow \sigma_{i}^{\tau / t}
$$


The estimation and normalization procedures proposed by Dayhoff et al. (1978) and extended by Jones et al. (1992) are essentially discrete time approximations of this process.

\subsection{A Heterogeneous Rates Model}

To model the evolution of a sequence of sites where the rate of evolution may vary from site to site, we propose the following generalization of the Markov model (also see Kelly 1991, Kelly and Rice 1994, Yang 1993). Let $\lambda_{h}$ be a multiplicative rate factor associated with site $h$ in the sequence $h=1, \ldots, n$. We assume that $\lambda_{h}$ are independent and identically distributed realizations of a random variable $\Lambda$. For identifiability, we assume the distribution of $\Lambda$ has mean one. The evolution at site $h$ may now be described as a Markov process with transition matrix

$$
\begin{aligned}
\mathbf{P}^{(h)}(t) & =e^{\mathbf{Q} t \lambda_{h}} \\
& =\mathbf{U}^{-1} e^{\Sigma t \lambda_{h}} \mathbf{U}
\end{aligned}
$$

An "average" replacement matrix that describes the mean behavior across many sites can be obtained by taking the expectation of $\mathbf{P}$ with respect to $\Lambda$,

$$
\begin{aligned}
E_{\Lambda} \mathbf{P}(t) & =\mathbf{U}^{-1} E_{\Lambda}\left(e^{\Sigma t \lambda_{h}}\right) \mathbf{U} \\
& =\mathbf{U}^{-1} \Phi(t) \mathbf{U}
\end{aligned}
$$

where

$$
\Phi(t)=\operatorname{diag}\left\{\phi\left(\sigma_{1} t\right), \ldots, \phi\left(\sigma_{20} t\right)\right\}
$$

and $\phi(x)$ is the moment generating function of $\Lambda$ evaluated at $x$ (Kelly and Rice 1994). 
Under this model, a replacement matrix can be adjusted to a time $\tau$ by the mapping

$$
\sigma_{i} \rightarrow \phi\left(\frac{\tau}{t} \phi^{-1}\left(\sigma_{i}\right)\right)
$$

For example, if the rates are gamma distributed with both scale and shape factors equal to $\alpha$ (this gives us a mean rate of one), then the mapping is

$$
\left.\sigma_{i} \rightarrow\left(1-\frac{\tau}{t}\left(1-\sigma_{i}^{\alpha}\right)\right)\right)^{-\alpha}
$$

\subsection{Alignment Scores}

Under the Markov model of evolution described above, the divergence time $t$ measures the similarity of two protein sequences. An alternative measure used in sequence comparison is the alignment score, $S$. The calculation of an alignment score depends on assigning similarity scores, $s_{i j}$, to each pair of amino acids $(i, j)$. The total score for two protein sequences is then just the sum of scores for each pair of amino acids in the sequence. Karlin and Altchul (1990) suggest scores based on the following log-odds ratio:

$$
s_{i j}=\log _{\lambda}\left(\frac{q_{i j}}{\pi_{i} \pi_{j}}\right)
$$

which is the $\log$ (base $\lambda$ ) odds of the pair occuring by evolution as opposed to the pair occuring by chance. Notice that the probability of the pair occuring by evolution is

$$
q_{i j}=\pi_{i} p_{i j}(t)
$$

where $\left\{\pi_{i}, i=1,20\right\}$ are the amino acid frequencies. This method of calculating alignment scores thus requires a replacement matrix $\mathbf{P}(t)$. 


\section{Results}

\subsection{Bias in Replacement Matrices}

If there is rate heterogeneity across the sites in a sequence, then adjusting a PAM matrix to time $t$ by multiplying the PAM1 matrix $t$ times will introduce a bias in the resulting replacement matrix. From Section 2.2, the average PAM1 matrix, assuming heterogeneous rates, can be written

$$
E_{\Lambda} \mathbf{P}(1)=\mathbf{U}^{-1} \boldsymbol{\Phi}(1) \mathbf{U}
$$

Assuming homogeneous rates, a replacement matrix $\mathbf{P}(t)$ is obtained by multipling out the PAM1 matrix $t$ times. When rates vary across the positions in a sequence, this method of adjustment introduces the following bias.

$$
\begin{aligned}
\text { bias } & =\mathbf{P}(1)^{t}-\mathbf{P}(t) \\
& =\mathbf{U}^{-1}\left(\boldsymbol{\Phi}(1)^{t}-\boldsymbol{\Phi}(t)\right) \mathbf{U}
\end{aligned}
$$

The diagonal matrix $\mathbf{\Phi}(1)^{t}-\boldsymbol{\Phi}(t)$ has entries $\varphi\left(\sigma_{i}\right)^{t}-\varphi\left(\sigma_{i} t\right)$. Jensen's inequality implies these eigenvalues will be underestimated when $t>1$ and overestimated when $t<1$.

$$
\begin{aligned}
& \varphi\left(\sigma_{i}\right)^{t}-\varphi\left(\sigma_{i} t\right)=E_{\Lambda}\left(\exp \left(\Lambda \sigma_{i}\right)\right)^{t}-E_{\Lambda}\left(\exp \left(\Lambda \sigma_{i} t\right)\right) \\
& \begin{cases}\leq E_{\Lambda}\left(\exp \left(\Lambda \sigma_{i} t\right)\right)-E_{\Lambda}\left(\exp \left(\Lambda \sigma_{i} t\right)\right)=0 & \text { if } t>1 \\
\geq E_{\Lambda}\left(\exp \left(\Lambda \sigma_{i} t\right)\right)-E_{\Lambda}\left(\exp \left(\Lambda \sigma_{i} t\right)\right)=0 & \text { if } t<1\end{cases}
\end{aligned}
$$

Equality holds above only when the rates are homogeneous.

An overall measure of the difference between the two replacement matrices is Barry and Hartigan's measure of distance $d=-1 / 20 \log (\operatorname{det}(\mathbf{P}(t)))$. The bias in this distance is

$$
\operatorname{bias}(d)=-1 / 20\left(\log \left(\operatorname{det}\left(\mathbf{P}(1)^{t}\right)\right)-\log (\operatorname{det}(\mathbf{P}(t)))\right)
$$




$$
\begin{aligned}
& =-1 / 20 \sum_{i=1}^{20}\left(\log \left(\varphi\left(\sigma_{i}\right)^{t}\right)-\log \left(\varphi\left(\sigma_{i} t\right)\right)\right. \\
& =-1 / 20 \sum_{i=1}^{20}\left(t \log \left(\varphi\left(\sigma_{i}\right)\right)-\log \left(\varphi\left(\sigma_{i} t\right)\right) .\right.
\end{aligned}
$$

As in Kelly and Rice (1994), a Taylor's series expansion gives an expression for this bias:

$$
\begin{aligned}
\operatorname{bias}(d) & =-1 / 20 \sum_{i=1}^{20} \sum_{n=1}^{\infty}\left(\frac{K_{n} t\left(\sigma_{i}\right)^{n}}{n !}-\frac{K_{n}\left(\sigma_{i} t\right)^{n}}{n !}\right) \\
& =-1 / 20 \sum_{i=1}^{20} \sum_{n=2}^{\infty} \frac{K_{n}\left(t-t^{n}\right)\left(\sigma_{i}\right)^{n}}{n !} \\
& =1 / 40 \operatorname{Var}(\Lambda)\left(t^{2}-t\right) \operatorname{tr}\left(\mathbf{Q}^{2}\right)+1 / 120 K_{3}\left(t^{3}-t\right) \operatorname{tr}\left(\mathbf{Q}^{3}\right)+\ldots
\end{aligned}
$$

Thus when $t>1$ the bias in distance is positive and increases with increasing time and increasing rate variability. When $t<1$, the bias is negative and increases with decreasing time and increasing rate variability.

If the rates have a Gamma distribution with parameter $\alpha$, the cumulants are $K_{n}=(n-1) ! \alpha^{1-n}$ (cf. Kendall p.91), and the bias simplifies to

$$
\operatorname{bias}(d)=-1 / 20 \sum_{i=1}^{20} \sum_{n=1}^{\infty} \alpha \frac{\left(t-t^{n}\right)\left(\sigma_{i} / \alpha\right)^{n}}{n}
$$

Figures 1 and 2 plot this bias (as a percentage of the Barry and Hartigan distance) against $t$ for various values of $\alpha$ using eigenvalues estimated from Henikoff and Henikoff's (1991) data (see Section 4.1).

\subsection{Bias in Alignment Score}

Because the alignment scores are functions of the replacement probabilities, they will also be biased when heterogeneous rates are not accounted for. In this section we illustrate the bias in the expected total alignment score for sequences of length $n$. Suppose that two sequences are produced by evolution 
according to the heterogeneous rate model with $\operatorname{gamma}(\alpha)$ distributed rates and divergence time $t$, then the expected data matrix is

$$
E(\mathbf{N})=n \mathbf{D P}(t, \alpha)
$$

where $\mathbf{D}$ is a diagonal matrix with entries $\pi_{i}$ and $\mathbf{P}(t, \alpha)$ is the average replacement matrix calculated from Equation (2). Suppose also that a divergence time $\hat{t}_{\text {homo }}$ is estimated assuming the homogeneous rate model using the method of maximum likelihood, and that a total alignment score, $\hat{S}$, is calculated for the sequences using $\mathbf{P}\left(\hat{t}_{\text {homo }}\right)$. Then the total score will have the following bias on average:

$$
\begin{aligned}
\operatorname{bias}(\hat{S}) & =\sum_{i, j=1}^{20} E\left(N_{i j}\right) \log \left(\frac{p_{i j}\left(\hat{t}_{h o m o}\right)}{\pi_{j}}\right)-\sum_{i, j=1}^{20} E\left(N_{i j}\right) \log \left(\frac{p_{i j}(t, \alpha)}{\pi_{j}}\right) \\
& =\sum_{i, j=1}^{20} E\left(N_{i j}\right) \log \left(\frac{p_{i j}\left(\hat{t}_{h o m o}\right)}{p_{i j}(t, \alpha)}\right) .
\end{aligned}
$$

The bias in the average alignment scores for a sequence of length $n=1000$ with $\alpha=1$ is plotted in Figure 3 . 


\section{Example}

In this section we illustrate the bias problems described above using Henikoff's blocks database Version 8.0 (Henikoff and Henikoff (1991)). This database contains 2880 blocks of aligned protein sequences from a number of species.

\subsection{Bias in the Replacement Matrix}

Using pairs of sequences with more than $85 \%$ similarity, we estimated a PAM1 matrix using the method described in Jones et al. (1992). This matrix is shown in Table 1. Tables 2 and 3 compare the PAM250 matrix calculated assuming a homogeneous rate model to the PAM250 matrix calculated assuming a heterogeneous rate model with a gamma rate distribution $(\alpha=1)$. The most noticable difference between these matrices is the overall rate of replacement. The probability of replacing any amino acid for another is the probability of observing amino acid $i$ multiplied by the probability that amino acid $i$ does not change:

$$
\sum_{i} \pi_{i}\left(1-p_{i i}(t)\right)
$$

For the homogeneous rate model replacement matrix this probability is .7819; for the heterogeneous rate model replacement matrix the probability is .6281 . In order to compare replacement matrices with equal rates of change, we determined the divergence time $t$ in the heterogeneous rate model (with $\alpha=1$ that yielded a probability of change $=.7819$; this time is $t=658$ PAMs. In comparing $\mathbf{P}(658,1)$ to the PAM250 matrix, we note that rare replacements (those with zero entries in the PAM1 matrix) are much more likely under the heterogeneous rate model. Results obtained with larger values of $\alpha$ are similar in nature but more extreme. 


\subsection{Testing for Heterogeneous Rates}

As discussed in Section 3, the severity of the biases encountered depends not only on whether rates are heterogeneous, but also on the extent of the variability. To illustrate that rates are typically heterogenous with a variability that will cause significant biases, we considered a subset of the blocks database. We fit both models to concatenated block sequences from E. coli and Homo sapiens from the Henikoff and Henikoff data set. The total length of the concatenated sequence was 958 amino acids, and the observed proportion of changes was .60. We estimated the divergence times for the two models, $t_{\text {homo }}$ and $t_{\text {heter }}$ and the heterogeneous rate model parameter $\alpha$ using the method of maximum likelihood. Maximizing the likelihood is equivalent to minimizing the goodness of fit statistic

$$
\chi_{\text {homo }}^{2}=2 \sum_{i=1}^{20} \sum_{j=1}^{20} N_{i j} * \log \left(\frac{N_{i j}}{n \pi_{i} P_{i j}(t)}\right) .
$$

in the homogeneous rate model, and

$$
\chi_{\text {heter }}^{2}=2 \sum_{i=1}^{20} \sum_{j=1}^{20} N_{i j} * \log \left(\frac{N_{i j}}{n \pi_{i} P_{i j}(t, \alpha)}\right) .
$$

in the heterogeneous rate model. Here $n$ is the length of the sequence, $\left\{\pi_{i}, i=\right.$ $1, \ldots, 20\}$ are the amino acid frequencies, and $\mathbf{P}(t)$ and $\mathbf{P}(t, \alpha)$ are the replacement matrices in the homogeneous rate and heterogeneous rate models, respectively.

For the E.coli - Homo sapiens data set:

$$
\begin{aligned}
& t_{\text {homo }}=137.66 \quad \chi_{\text {homo }}^{2}=486.8 \\
& t_{\text {heter }}=276.61 \quad \chi_{\text {heter }}^{2}=412.9 \\
& \alpha=1.432
\end{aligned}
$$


The difference in the chi-square statistics is

$$
\chi_{\text {homo }}^{2}-\chi_{\text {heter }}^{2}=73.9
$$

which is highly significant if the statistic is chi-square distributed with one degree of freedom.

The sparseness of the raw data matrix $(\mathbf{N})$, may make the chi-square approximation to statistic (12) questionable (see for example Goldman (1993)). To assess the distribution of this statistic, a parametric bootstrap confidence interval was calculated in the following way (also described in Goldman (1993)). To test

$H_{0}:$ rates are homogeneous

versus

$H_{A}:$ rates are heterogeneous

we simulated one thousand data matrices, or one thousand multinomial random variables with parameters $n=958$ and $\left.\mathbf{p}=\mathbf{D P}\left(t_{\text {homo }}\right)\right)$. For each bootstrap matrix, the difference in chi-square statistics (12) is calculated. A histogram of this statistic is shown in Figure 4. An upper $95^{\text {th }}$ percentile of 2.80 and an upper $99^{\text {th }}$ percentile of 5.68 was obtained from the bootstrapped statistics. This provides significant evidence that the heterogeneous rate model with $\alpha=1.432$ fits significantly better than the homogeneous rate model.

\subsection{Bias in Alignment Scores}

In this section we illustrate the biases in alignment scores that occur when heterogeneous rates are ignored using the concatenated Homo sapiens and E.coli sequences described above. We calculated the total alignment score for this pair of sequences for various times first using the homogeneous rate 
replacement matrix and then using a heterogenous rate replacement matrix assuming gamma distributed rates with $\alpha=1.432$. These scores are displayed in Figure 5. As in the previous analysis, the homogeneous rate model suggests a divergence time of approximately 138 PAMs (which gives a maximum score of 536.6); whereas the heterogenous rate model suggests a divergence time of approximately 277 PAMs (which gives a maximum score of 573.5). Certainly, estimating divergence time with alignment scores will introduce a significant bias if heterogeneous rates are not considered. 


\section{Discussion}

In an exhaustive matching of the entire protein sequence database, Gonnet et al. made the intriguing observation that "mutation matrices (normalized to a distance of 250 PAM ...) were found to differ, depending on whether they were derived from protein pairs that are distantly homologous or from protein pairs that are closely homologous." This observation may be interpreted as giving evidence that the PAM matrix is inadequate for aligning distantly related proteins. Results presented here, however, provide an alternate explanation for these findings. We demonstrate that the bias in normalizing a mutation matrix to 1 PAM may be positive or negative depending on whether the distance between the proteins is larger or smaller than 1. Gonnet et al. normalized to 250 PAMs, but the results are qualitatively equivalent: distantly homologous protein pairs $(t>250$ PAMs) will have a positive bias and closely homogous pairs ( $t<250$ PAMs) will have a negative bias.

We note that Yang (1993) has found a heterogeneous rate model with $\alpha=4$ provides a good fit to nucleotide sequences. Our results suggest that there is less rate variability in amino acid sequences $(\alpha<2)$ than in nucleotide sequences. This result might be expected since the amino acid model does not have to account for the rate differences in different codon positions. - Estimates of rate variation in amino acid repalcements obtained by other methods (Uzzell and Corbin 1971, Ota and Nei 1994) suggest a value of approximately $\alpha=2$. The true value of $\alpha$ will depend on the particular set of sequences under study. Thus it may not be possible to obtain a definitive value of $\alpha$ that is applicable to all amino acid sequences.

The results presented here demonstrate that there is a bias introduced into estimated replacement matrices due to rate heterogeneity. A method for 
correctly extrapolating replacement matrices estimated from closely related sequences to matrices appropriate for longer divergence times is provided. The evolution of protein sequences is a complex process and the methods described here are based on a number of simplifying assumptions. For example, the mutational spectrum as defined by the rate matrix $\mathbf{Q}$ in Equation (1) is likely to vary from site to site. This leads us to ask, does it make sense to estimate an average replacement matrix? For many practical purposes, such as database searching and sequence alignment, scoring methods based on average replacement matrices have proven to be very effective. We are hopeful that the bias correction proposed here will serve to improve their utility. 


\section{References}

Altschul, S.F. 1991. Amino acid substitution matrices from an information theoretic perspective. J. Mol. Biol. 219, 555-565.

Altschul, S.F. 1993. A protein alignmnet scoring system sensitive at all evolutionary distances. J. Mol. Evol. 36, 290-300.

Altschul, S.F., Gish, W., Webb, M., Myers, E.W., Lipman, D.J. 1990. Basic local alignment search tool. J. Mol. Biol. 215,403-410.

Brown M., Hughey R., Krogh A., Mian I.S., Sjölander K., Haussler D. 1993. Using Dirichlet mixture priors to derive hidden Markov models for protein families. In Proceedings of the 1st International Conference on Intelligent Systems for Molecular Biology.

Dayhoff M.O., Schwartz R.M., Orcutt B.C. 1978. A model of evolutionary change in proteins, pp. 345-352. In Atlas of protein sequence structure, vol. 5, suppl. 3 M.O. Dayhoff (ed.., National Biomedical Research Foundation, Washington D.C.

Feng D.A., Doolittle R. 1985. Aligning amino acid sequences, comparison of commonly used methods. J. Mol. Evol. 21, 112-125.

Goldman N. 1993. Statistical tests of models of DNA substitution J. Mol. Evol. 36, 182-198.

Gonnet G.H., Cohen M.A., Benner S.A. 1992. Exhaustive matching of the entire protein sequence database. Science 256, 1443-1445.

Henikoff, S., Henikoff, J.G. 1991. Automated assembly of protein blocks for database searching. Nucleic Acids Res 19, 6565-6572. 
Holmquist, R., Goodman, M., Conroy, T., Czelusniak, J. 1983. The spatial distribution of fixed mutations within genes coding for proteins. J. Mol. Evol. $19,437-448$.

Jones, D.T., Taylor, W.R., Thornton, J.M. 1992. The rapid generation of mutation data matrices from protein sequences. CABIOS 8, 275-282.

Karlin, S., Altschul, S.F. 1990. Methods for assessing the statistical significance of molecular sequence features by using general scoring schemes. Proc. Natl. Acad. Sci. USA 87, 2264-2268.

Kelly, C. 1991. PhD dissertation, UC San Diego

Kelly, C., Rice, J. 1994. Modeling molecular evolution: a heterogeneous rate analysis. Mathematical Biosciences (to appear)

Ota, T., Nei, M. 1994. Estimation of the number of amino acid substitutions per site when the substitution rate varies across site. J. Mol. Evol. 38, 642643.

Reeves, J.A. 1992. Heterogeneity in the substitution process of amino acid sites of proteins coded for by mitochondrial DNA J. Mol. Evol. 35, 17-31.

Sander, C., Schnieder, R. 1991. Database homology-derived protein structures and the structural meaning of sequence alignment. Proteins 9, 56-68.

Schwartz, R.M., Dayhoff, M.O. 1978. Matrices for detecting distant relationships, pp/353-358 in Atlas of protein sequence structure, vol. 5, suppl. 3 M.O. Dayhoff (ed.., National Biomedical Research Foundation, Washington D.C.

Stuart, A., Ord, J.K. 1987. Kendall's Advanced Theory of Statistics. Fifth Edition, Vol 1, Oxford Univeristy Press, New York 
Taylor, W.R. 1986. The classification of amino acid conservation J. Theor. Biol. 119, 205-218.

Uzzell, T., Corbin, K.W. 1971. Fitting discrete probability distributions to evolutionary events. Science 172, 1089-1096.

Wakeley, J. 1993. Substitution rate variation among sites in hypervariable region 1 if human mitchondrial DNA J. Mol Evol. 37, 613-623.

Wilbur, J.W. 1985. On the PAM matrix model of protein evolution. Mol. Biol. Evol. 2, 434-447.

Yang, Z. 1993. Maximum-likelihood estimation of phylogeny from DNA sequences when substitution rates differ over sites. Mol. Biol. Evol. 10, 13961401.

Yang, Z., Goldman, N., Friday, A. 1994. Comparison of models for nucleotide substitution used in maximum-likelihood phylogenetic inference. Mol. Biol. Evol. 11, 316-324. 


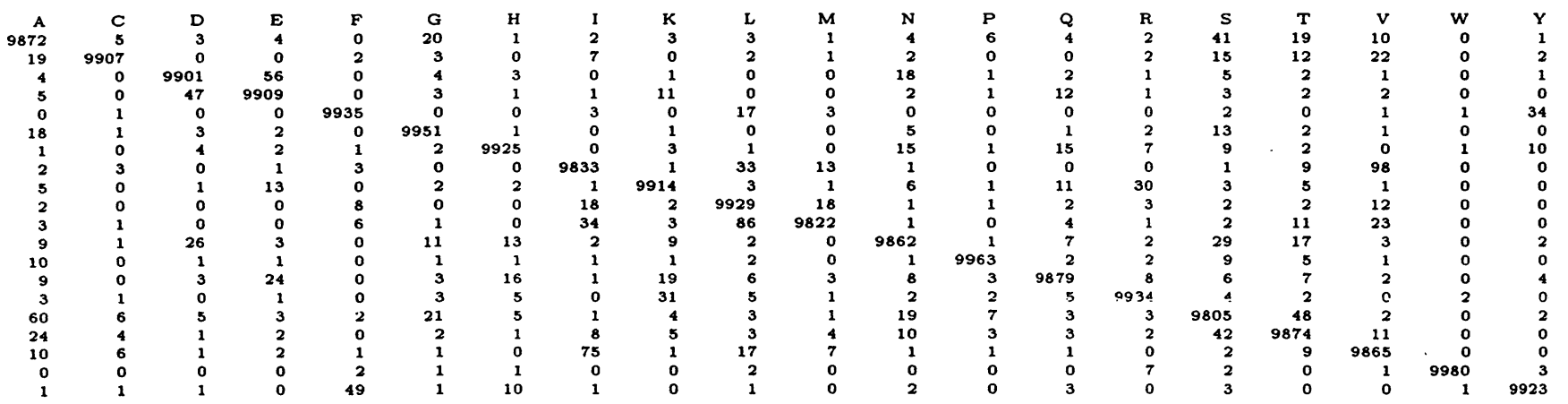

Table 1: PAM1 Matrix estimated using Henikoff and Henikoff's data. 


\begin{tabular}{|c|c|c|c|c|c|c|c|c|c|c|c|c|c|c|c|c|c|c|c|}
\hline $\mathbf{A}$ & C & D & E & $\mathbf{F}$ & G & H & I & $\mathrm{K}$ & L & $\mathbf{M}$ & $\mathbf{N}$ & $P$ & $\mathbf{Q}$ & $\mathbf{R}$ & $s$ & $\mathbf{T}$ & $\mathrm{v}$ & w & $\mathbf{Y}$ \\
\hline 1374 & 288 & 433 & 491 & 171 & 1293 & 245 & 432 & 424 & 605 & 150 & 418 & 519 & 271 & 351 & 831 & 899 & 633 & 37 & 137 \\
\hline 1039 & 1194 & 240 & 268 & 311 & 733 & 183 & 744 & 290 & 862 & 211 & 312 & 260 & 182 & 298 & 683 & 835 & 1092 & 52 & 212 \\
\hline 658 & 101 & 2011 & 2032 & 101 & 719 & 342 & 184 & 562 & 273 & 70 & 606 & 208 & 416 & 315 & 496 & 479 & 280 & 21 & 123 \\
\hline 623 & 94 & 1699 & 2297 & 91 & 626 & 305 & 198 & 749 & 305 & 78 & 481 & 209 & 513 & 418 & 444 & 441 & 298 & 23 & 107 \\
\hline 280 & 141 & 109 & 117 & 3139 & 190 & 288 & 470 & 136 & 1411 & 247 & 144 & 95 & 150 & 143 & 241 & 249 & 519 & 128 & 1804 \\
\hline 1159 & 182 & 424 & 442 & 104 & 3515 & 214 & 212 & 334 & 310 & 81 & 409 & 271 & 216 & 306 & 751 & 607 & 335 & 32 & 97 \\
\hline 582 & 121 & 535 & 571 & 417 & 566 & 1875 & 199 & 572 & 416 & 94 & 574 & 280 & 583 & 644 & 526 & 487 & 274 & 97 & 588 \\
\hline 594 & 284 & 166 & 215 & 395 & 325 & 115 & 1444 & 229 & 2026 & 445 & 195 & 188 & 152 & 208 & 376 & 605 & 1832 & 38 & 169 \\
\hline 608 & 115 & 530 & 846 & 119 & 533 & 345 & 239 & 1928 & 524 & 119 & 388 & 265 & 506 & 1463 & 446 & 509 & 322 & 77 & 118 \\
\hline 445 & 176 & 132 & 176 & 633 & 254 & 129 & 1082 & 269 & 3048 & 528 & 159 & 182 & 170 & 296 & 295 & 443 & 1259 & 61 & 261 \\
\hline 529 & 206 & 163 & 216 & 530 & 318 & 140 & 1139 & 292 & 2530 & 557 & 190 & 181 & 189 & 281 & 353 & 547 & 1368 & 49 & 224 \\
\hline 902 & 187 & 861 & 818 & 189 & 985 & 522 & 307 & 584 & 467 & 117 & 767 & 309 & 375 & 438 & 712 & 772 & 439 & 40 & 210 \\
\hline 893 & 124 & 236 & 284 & 99 & 520 & 203 & 235 & 318 & 427 & 88 & 246 & 4100 & 234 & 311 & 618 & 615 & 344 & 21 & 80 \\
\hline 687 & 128 & 695 & 1025 & 232 & 612 & 623 & 280 & 896 & 589 & 136 & 441 & 345 & 849 & 713 & 503 & 549 & 382 & 32 & 261 \\
\hline 522 & 123 & 309 & 490 & 130 & 509 & 404 & 225 & 1521 & 600 & 119 & 302 & 269 & 418 & 2587 & 395 & 418 & 294 & 237 & 126 \\
\hline 1198 & 273 & 471 & 504 & 212 & 1207 & 319 & 394 & 449 & 580 & 144 & 475 & 517 & 286 & 382 & 839 & 950 & 567 & 52 & 180 \\
\hline 1130 & 291 & 397 & 437 & 191 & 851 & 258 & 553 & 446 & 758 & 195 & 450 & 449 & 272 & 353 & 829 & 1199 & 766 & 35 & 141 \\
\hline 66.5 & 319 & 194 & 247 & 3.33 & 39.3 & 121 & $14 n 1$ & 2.35 & 1201 & Ang & 214 & 210 & $15 \Omega$ & 208 & 41.3 & 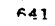 & 1855 & 36 & 145 \\
\hline 176 & 69 & 68 & 87 & 373 & 174 & 195 & 131 & 258 & 400 & 67 & 89 & 60 & 99 & 764 & 173 & 132 & 164 & 6163 & 359 \\
\hline 320 & 137 & 189 & 197 & 2568 & 253 & 577 & 287 & 191 & 829 & 149 & 226 & 109 & 240 & 197 & 291 & 261 & 323 & 175 & 2481 \\
\hline
\end{tabular}

Table 2: PAM250 Matrix - Estimated Assuming Homogeneous Rates. 


\begin{tabular}{|c|c|c|c|c|c|c|c|c|c|c|c|c|c|c|c|c|c|c|c|}
\hline $\mathbf{A}$ & C & D & $E$ & $\mathbf{F}$ & G & H & I & K & L & $\mathbf{M}$ & $\mathbf{N}$ & $\mathbf{P}$ & $\mathbf{Q}$ & $\mathbf{R}$ & $s$ & $\mathbf{T}$ & $\mathbf{v}$ & $\mathbf{w}$ & $Y$ \\
\hline 3033 & 233 & 327 & 381 & 145 & 999 & 180 & 323 & 329 & 475 & 116 & 320 & 396 & 216 & 271 & 837 & 755 & 513 & 34 & 115 \\
\hline 841 & 3150 & 189 & 216 & 247 & 535 & 142 & 553 & 224 & 622 & 150 & 234 & 196 & 138 & 244 & 566 & 650 & 885 & 46 & 173 \\
\hline 496 & 80 & 3606 & 1703 & 100 & 545 & 253 & 160 & 393 & 258 & 61 & 536 & 172 & 289 & 242 & 385 & 355 & 237 & 24 & 104 \\
\hline 485 & 76 & 1424 & 3845 & 93 & 480 & 216 & 173 & 586 & 274 & 67 & 335 & 173 & 429 & 304 & 333 & 331 & 260 & 26 & 92 \\
\hline 238 & 112 & 108 & 120 & 4544 & 187 & 201 & 360 & 128 & 1078 & 189 & 117 & 94 & 117 & 131 & 207 & 206 & 392 & 99 & 1372 \\
\hline 896 & 133 & 321 & 339 & 102 & 4919 & 163 & 177 & 259 & 283 & 70 & 311 & 209 & 165 & 245 & 590 & 426 & 272 & 31 & 87 \\
\hline 427 & 93 & 396 & 404 & 291 & 432 & 3703 & 172 & 405 & 353 & 79 & 491 & 219 & 496 & 486 & 427 & 357 & 233 & 77 & 459 \\
\hline 445 & 211 & 145 & 187 & 302 & 271 & 100 & 2827 & 190 & 1554 & 380 & 156 & 156 & 120 & 172 & 281 & 481 & 1851 & 36 & 137 \\
\hline 472 & 89 & 371 & 661 & 112 & 414 & 245 & 198 & 3665 & 426 & 99 & 312 & 209 & $\$ 16$ & 1144 & 343 & 401 & 263 & 61 & 100 \\
\hline 349 & 127 & 125 & 159 & 483 & 232 & 109 & 831 & 219 & 4504 & 472 & 131 & 156 & 142 & 242 & 236 & 332 & 909 & 53 & 191 \\
\hline 409 & 147 & 142 & 185 & 406 & 273 & 117 & 973 & 244 & 2262 & 2127 & 151 & 148 & 168 & 224 & 277 & 464 & 1072 & 43 & 168 \\
\hline 690 & 140 & 762 & 570 & 155 & 750 & 446 & 245 & 470 & 384 & 93 & 2548 & 232 & 301 & 325 & 677 & 661 & 346 & 37 & 170 \\
\hline 682 & 94 & 195 & 234 & 99 & 401 & 159 & 195 & 251 & 365 & 73 & 185 & 5315 & 185 & 248 & 483 & 458 & 281 & 24 & 76 \\
\hline 549 & 97 & 482 & 857 & 181 & 468 & 530 & 221 & 737 & 490 & 121 & 354 & 272 & 2717 & 532 & 393 & 435 & 305 & 45 & 212 \\
\hline 404 & 101 & 237 & 356 & 119 & 408 & 305 & 187 & 1189 & 490 & 95 & 224 & 214 & 312 & 4204 & 310 & 318 & 244 & 176 & 106 \\
\hline 1200 & 226 & 365 & 378 & 182 & 949 & 259 & 295 & 345 & 463 & 113 & 452 & 404 & 223 & 300 & 2253 & 965 & 424 & 47 & 150 \\
\hline 949 & 227 & 294 & 328 & 158 & 598 & 189 & 439 & 352 & 369 & 166 & 385 & 334 & 215 & 268 & 842 & 2934 & ses & 33 & 115 \\
\hline 540 & 258 & 164 & 215 & 252 & 319 & 103 & 1415 & 193 & 1300 & 320 & 168 & 172 & 126 & 172 & 309 & 506 & 3310 & 35 & 121 \\
\hline 165 & 61 & 77 & 97 & 289 & 168 & 155 & 124 & 202 & 343 & 59 & 81 & 66 & 85 & 568 & 156 & 125 & 158 & 6748 & 274 \\
\hline 269 & 112 & 160 & 169 & 1953 & 226 & 451 & 233 & 163 & 607 & 111 & 183 & 103 & 194 & 166 & 243 & 214 & 269 & 133 & 4043 \\
\hline
\end{tabular}

Table 3: PAM250 Matrix - Estimated Assuming Heterogeneous Rates with $\alpha=1$ 


\begin{tabular}{|c|c|c|c|c|c|c|c|c|c|c|c|c|c|c|c|c|c|c|c|}
\hline A & C & D & $E$ & $\mathbf{F}$ & G & H & I & $\mathrm{K}$ & L & $\mathbf{M}$ & $\mathbf{N}$ & $\mathbf{P}$ & $\mathbf{Q}$ & $\mathbf{R}$ & $S$ & $T$ & $\mathbf{v}$ & $\mathbf{w}$ & $\mathbf{Y}$ \\
\hline 1818 & 233 & 425 & 499 & 264 & 1007 & 250 & 433 & 433 & 718 & 161 & 352 & 447 & 262 & 386 & 712 & 717 & 617 & 72 & 195 \\
\hline 839 & 1592 & 322 & 378 & 347 & 706 & 221 & 594 & 358 & 859 & 192 & 295 & 311 & 211 & 358 & 569 & 662 & 863 & 82 & 240 \\
\hline 645 & 136 & 2077 & 1332 & 219 & 728 & 305 & 305 & 501 & 532 & 118 & 482 & 291 & 325 & 381 & 471 & 485 & 421 & 63 & 182 \\
\hline 634 & 133 & 1113 & 2271 & 214 & 682 & 284 & 313 & 611 & 545 & 122 & 378 & 291 & 398 & 426 & 441 & 469 & 434 & 65 & 174 \\
\hline 433 & 158 & 236 & 276 & 2718 & 405 & 270 & 479 & 268 & $11 \mathrm{B1}$ & 221 & 206 & 207 & 190 & 267 & 328 & 363 & 571 & 139 & 1084 \\
\hline 902 & 176 & 429 & 482 & 222 & 3026 & 240 & 325 & 392 & 560 & 126 & 354 & 326 & 234 & 368 & 604 & 543 & 456 & 68 & 169 \\
\hline 594 & 145 & 476 & 531 & 392 & 635 & 1979 & 322 & 499 & 623 & 134 & 448 & 321 & 430 & 534 & 487 & 481 & 427 & 113 & 430 \\
\hline 596 & 227 & 276 & 339 & 402 & 499 & 186 & 1673 & 324 & 1478 & 334 & 237 & 270 & 194 & 305 & 395 & 554 & 1411 & 74 & 227 \\
\hline 620 & 143 & 472 & 690 & 235 & 627 & 301 & 337 & 2084 & 663 & 145 & 348 & 317 & 389 & 951 & 441 & 506 & 447 & 102 & 183 \\
\hline 528 & 175 & 257 & 316 & 530 & 459 & 193 & 790 & 340 & 2874 & $\mathbf{3 8 6}$ & 219 & 264 & 204 & 347 & 360 & 464 & 929 & 90 & 274 \\
\hline 568 & 188 & 274 & 337 & 474 & 494 & 198 & 855 & 357 & 1849 & 1080 & 234 & 264 & 220 & 339 & 388 & 537 & 1009 & 82 & 254 \\
\hline 760 & 177 & 684 & 643 & 271 & 851 & 407 & 373 & 525 & 643 & 143 & 1344 & 337 & 317 & 429 & 625 & 657 & 506 & 76 & 231 \\
\hline 769 & 148 & 329 & 394 & 218 & 626 & 233 & 338 & 381 & 619 & 129 & 269 & 3161 & 242 & 367 & 536 & 557 & 462 & 61 & 159 \\
\hline 665 & 149 & 543 & 796 & 294 & 663 & 459 & 357 & 688 & 706 & 158 & 373 & 357 & 1404 & 565 & 471 & 527 & 478 & 85 & 260 \\
\hline 374 & 148 & 374 & 199 & 242 & 612 & 335 & 330 & 988 & 704 & 144 & 296 & 318 & 332 & 2411 & 418 & 455 & 434 & 197 & 189 \\
\hline 1026 & 227 & 447 & 501 & 288 & 971 & 295 & 414 & 144 & 707 & 159 & 417 & 449 & 268 & 404 & 1228 & 815 & Est & 82 & $2 \mathrm{is}$ \\
\hline yul & 231 & 402 & 465 & 278 & 761 & 255 & 506 & 144 & 794 & 192 & 382 & 407 & 261 & 384 & 711 & 1676 & 682 & 72 & 198 \\
\hline 649 & 252 & 292 & 359 & 367 & 535 & 189 & 1079 & 328 & 1330 & 301 & 247 & 282 & 198 & 306 & 414 & 571 & 2016 & 73 & 213 \\
\hline 346 & 109 & 198 & 244 & 408 & 365 & 228 & 259 & 341 & 588 & 112 & 168 & 169 & 161 & 633 & 273 & 273 & 333 & 4447 & 344 \\
\hline 454 & 155 & 280 & 320 & 1542 & 440 & 423 & 384 & 298 & 870 & 168 & 249 & 216 & 239 & 297 & 352 & 367 & 473 & 167 & 2306 \\
\hline
\end{tabular}

Table 4: PAM658 Matrix - Estimated Assuming Heterogeneous Rates with $\alpha=1$ 


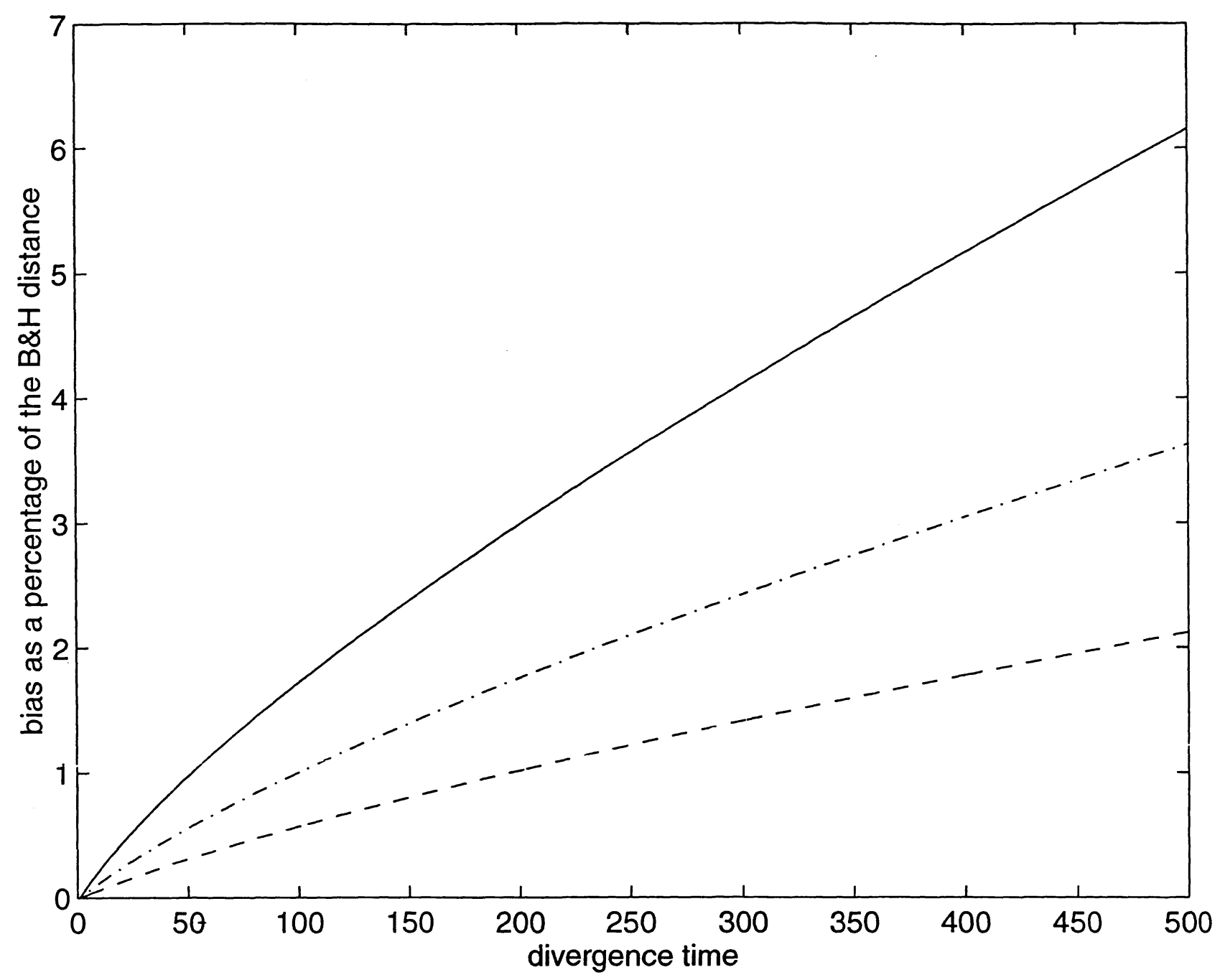

Figure 1: Bias in Barry and Hartigan's distance measure as a percentage of the distance for divergence times $>1$. A heterogeneous rate model with gamma distributed rates was assumed ( $\alpha=1:--, \alpha=2:-, \alpha=4:-)$. 


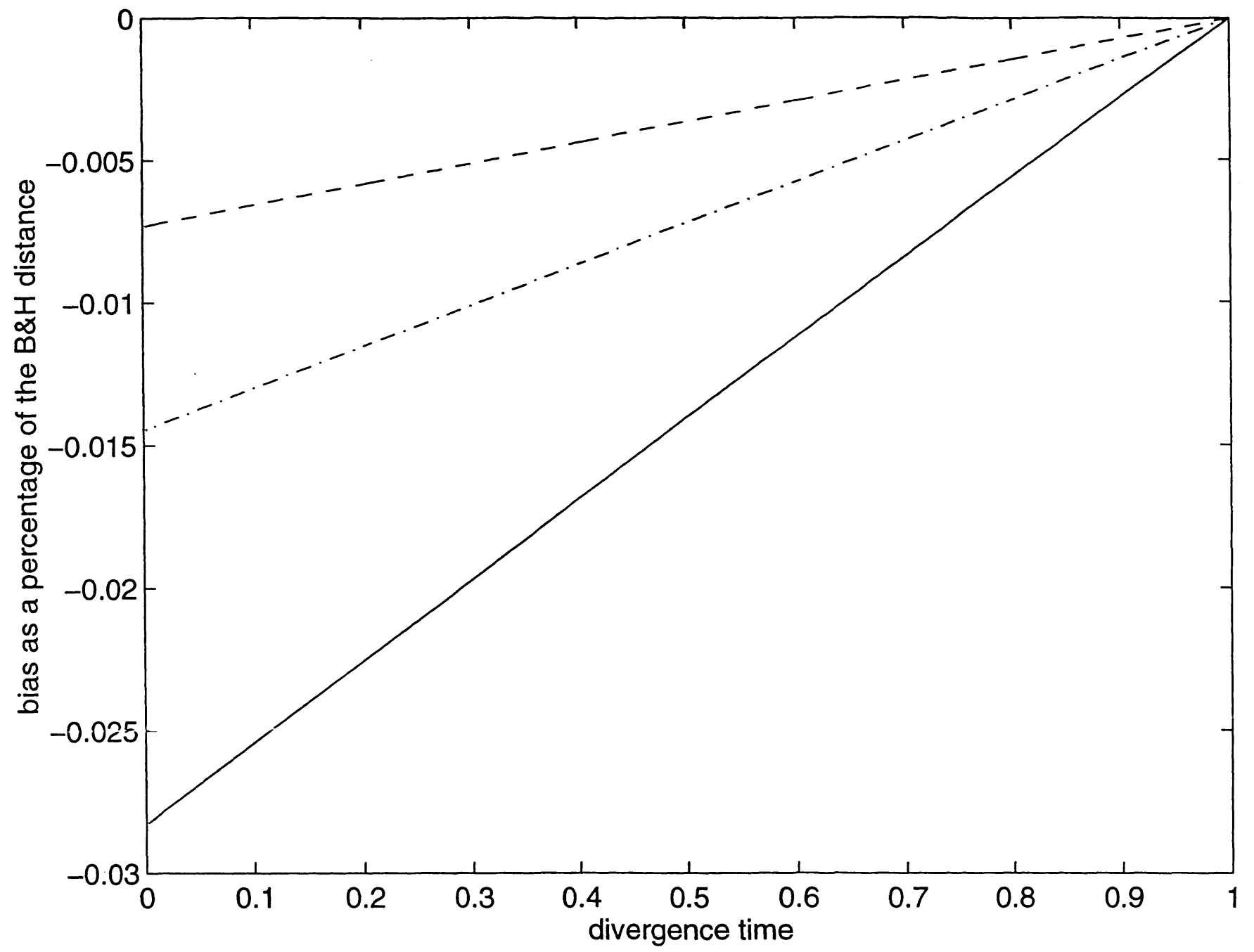

Figure 2: Bias in Barry and Hartigan's distance measure as a percentage of the distance for divergence times $<1$. A heterogeneous rate model with gamma distributed rates was assumed $(\alpha=1:--, \alpha=2:-, \alpha=4:-)$. 


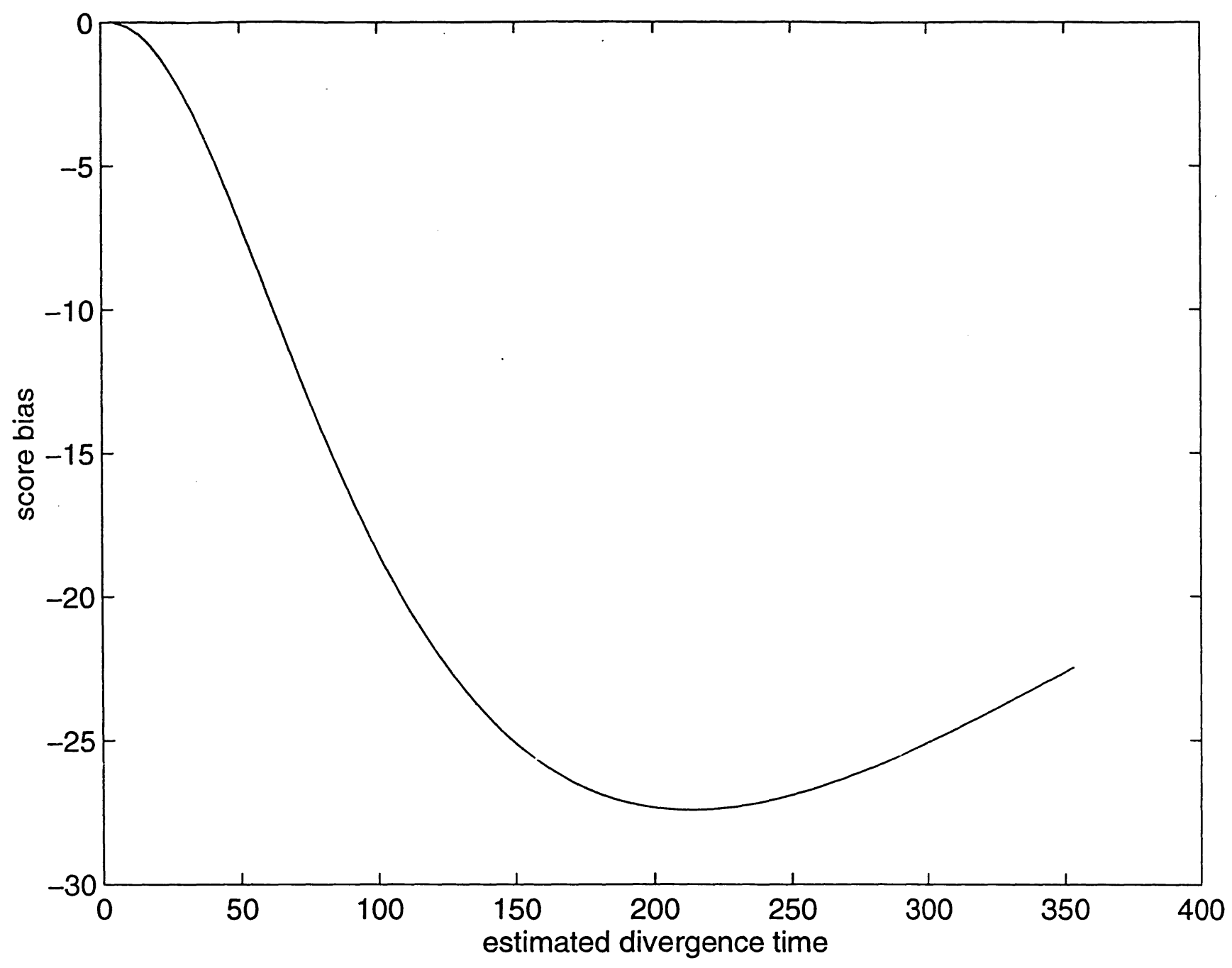

Figure 3: Bias in the expected alignment score for sequences of length $n=1000$ as a function of divergence time when rates have a gamma $(\alpha=1)$ distribution. 


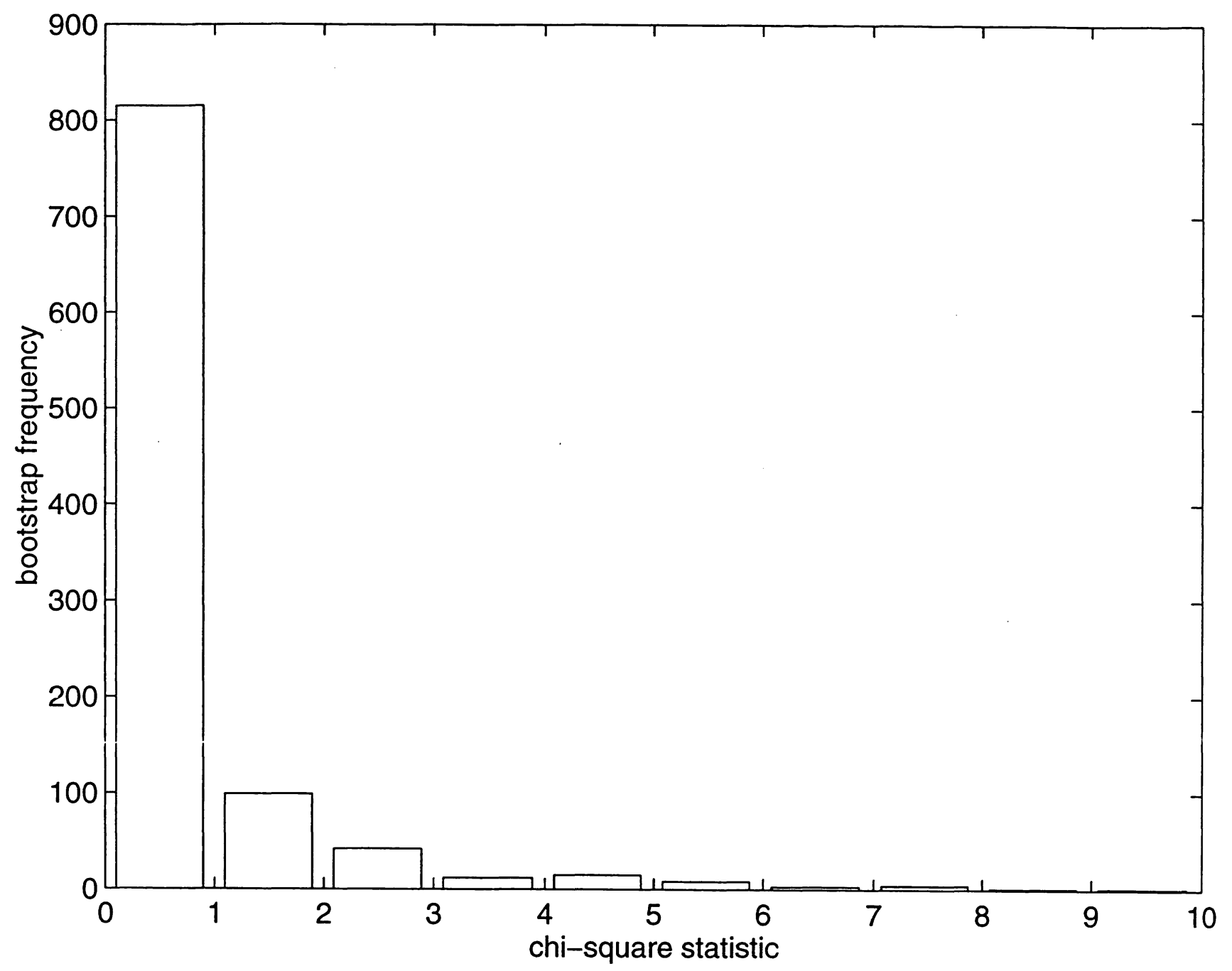

Figure 4: Histogram of bootstrapped chi-square statistics. 


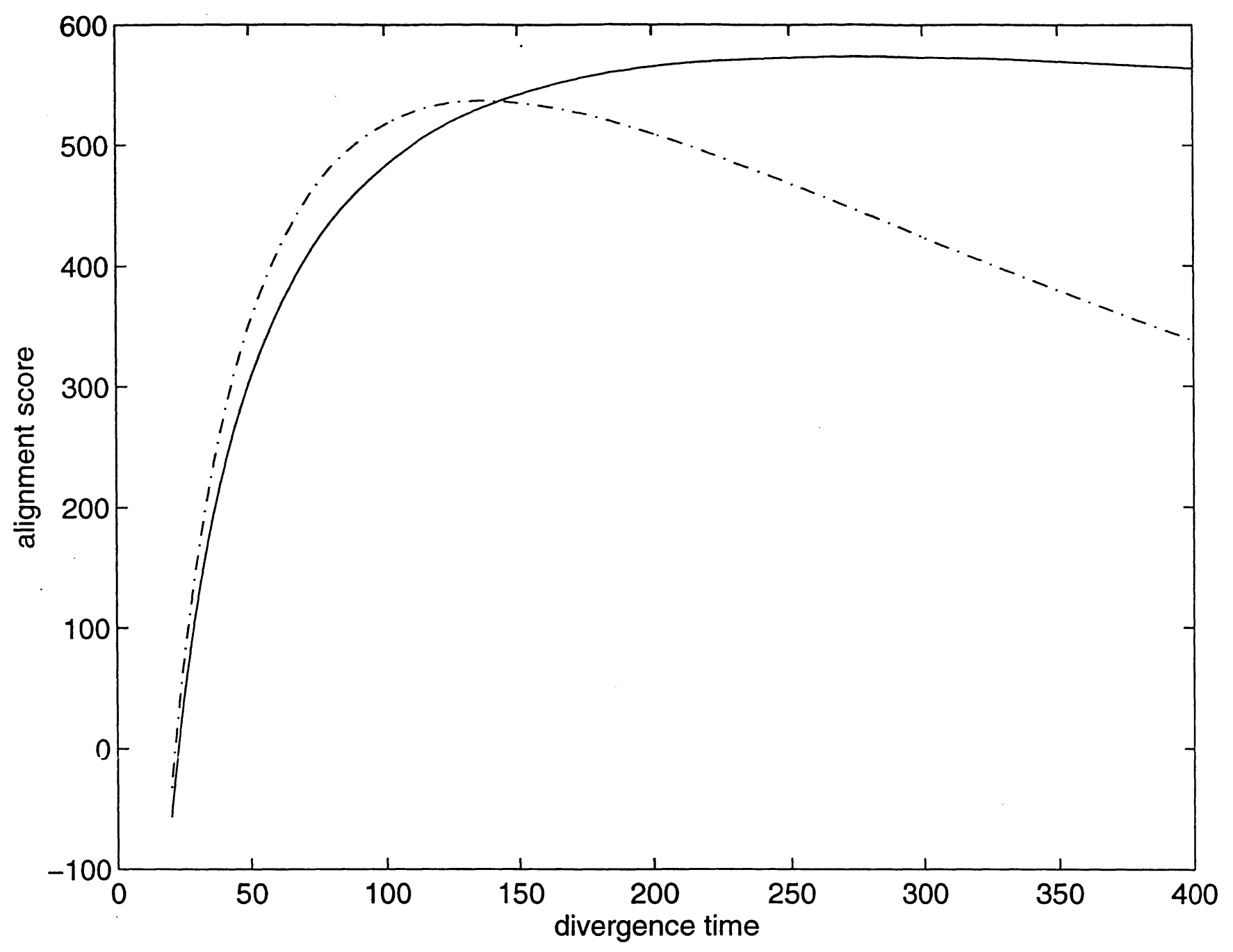

Figure 5: Alignment scores versus divergence time calculated for the Homo sapien - E.coli dataset assuming a homogeneous rate model ( - . ) and a heterogeneous rate model with $\alpha=1.432$ ( - ). 